\title{
An Upper Bound for the First Zero of Bessel Functions
}

\section{By L. G. Chambers}

Abstract. It is shown, using the Rayleigh-Ritz method of the calculus of variations, that an upper bound for the first zero $j_{\nu}$, of $z^{-\nu} J_{\nu}(z), \nu>-1$, is given by

$$
(\nu+1)^{1 / 2}\left\{(\nu+2)^{1 / 2}+1\right\} \text {, }
$$

and that for large $\nu, j_{\nu}=\nu+O\left(\nu^{1 / 2}\right)$.

1. The following upper bound is given by Watson [4] for the first zero $j_{\nu}$ of $J_{\nu}(x)$ $(\nu>0)$

$$
j_{\nu}<\left\{\frac{4}{3}(\nu+1)(\nu+5)\right\}^{1 / 2} .
$$

It may be shown that a better bound may be obtained, valid for $\nu>-1$, namely

$$
(\nu+1)^{1 / 2}\left((\nu+2)^{1 / 2}+1\right) \text {. }
$$

2. Consider the function

$$
u(z)=\Gamma(\nu+1)(2 /(\gamma z))^{\nu} J_{\nu}(\gamma z) .
$$

The differential equation satisfied by $u(z)$ is given by Watson [3] to be

$$
z^{2} u^{\prime \prime}+(2 \nu+1) z u^{\prime}+\gamma^{2} z^{2} u=0
$$

with the boundary condition $u(0)=1$, and if $\gamma$ is a zero of $J_{\nu}, u(1)=0$.

Equation (4) can be written in Sturm-Liouville form

$$
\frac{d}{d z}\left(z^{2 v+1} \frac{d u}{d z}\right)+\gamma^{2} z^{2 \nu+1} u=0 .
$$

Multiplying Eq. (5) by $u$ and integrating over $0 \leqslant z \leqslant 1$, it follows that

$$
\gamma^{2}=\frac{\int_{0}^{1} z^{2 \nu+1} u^{\prime 2} d z}{\int_{0}^{1} z^{2 \nu+1} u^{2} d x} .
$$

On integration by parts, $u u^{\prime} z^{2 \nu+1}$ will vanish at $z=0$, if $\nu>-\frac{1}{2}$, and $u(1)$ vanishes. Thus the relation (6) provides a variational formulation, as indicated by Irving and Mullineux [1], for $\gamma^{2}$ which is an eigenvalue for the differential equation (5). The first eigenvalue will be $j_{\nu}^{2}$. The functional

$$
\Lambda(\omega)=\frac{\int_{0}^{1} z^{2 \nu+1} \omega^{\prime 2} d z}{\int_{0}^{1} z^{2 \nu+1} \omega^{2} d z},
$$

Received February 19, 1981; revised July 13, 1981.

1980 Mathematics Subject Classification. Primary 33A40, 49G10. 
as indicated by Irving and Mullineux [2], obeys the following relations

$$
\begin{gathered}
\Lambda(u)=j_{\nu}^{2}, \\
\Lambda(\omega)>\Lambda(u), \quad u \neq \omega .
\end{gathered}
$$

Thus $\Lambda(\omega)$ provides an upper bound to $j_{\nu}^{2}$ when $\omega(1)=0$, by the Rayleigh-Ritz procedure.

3. Consider the approximating function

$$
\omega=1-z^{p}
$$

where $p$ is as yet unspecified.

$$
\begin{aligned}
\Lambda(\omega) & =\frac{\int_{0}^{1} z^{2 \nu+1} p^{2} z^{2 p-2} d z}{\int_{0}^{1}\left(1-2 z^{p}+z^{2 p}\right) z^{2 \nu+1} d z} \\
& =p^{2}\left\{\frac{1 /(2 \nu+2 p)}{1 /(2 \nu+2)-2 /(2 \nu+p+2)+1 /(2 \nu+2 p+2)}\right\} \\
& =\frac{(\nu+1)(2 \nu+p+2)(\nu+p+1)}{\nu+p}
\end{aligned}
$$

on simplification.

Up till now $p$ has not been specified. $\Lambda(\omega)$ may be regarded as a function of $p$, and the best upper bound will follow by minimizing $\Lambda(\omega)$ with respect to $p$. It can be verified by differentiation that this happens when

$$
p+\nu=(\nu+2)^{1 / 2} \text {. }
$$

Although $p$ is negative outside of $-1<v<2$, the process is still valid because all of the denominators in the expression (11) remain positive, and it can easily be seen that

$$
\operatorname{lt}_{z \rightarrow 0} z^{2 \nu+1} \omega \omega^{\prime}=0
$$

so that the endpoint condition at $z=0$ remains satisfied. It follows that

$$
j_{\nu}^{2}<\frac{(\nu+1)\left[(\nu+2)+(\nu+2)^{1 / 2}\right]\left[(\nu+2)^{1 / 2}+1\right]}{(\nu+2)^{1 / 2}}
$$

which gives

$$
j_{\nu}<(\nu+1)^{1 / 2}\left((\nu+2)^{1 / 2}+1\right) .
$$

A straightforward reduction shows that, if $\nu+1>0$,

$$
(\nu+1)^{1 / 2}\left((\nu+2)^{1 / 2}+1\right)<\left\{\frac{4}{3}(\nu+1)(\nu+5)\right\}^{1 / 2} .
$$

(For $\nu=7$ there is in fact equality.)

It thus follows that [4]

$$
\{\nu(\nu+2)\}^{1 / 2}<j_{\nu}<(\nu+1)^{1 / 2}\left((\nu+2)^{1 / 2}+1\right), \quad \nu>1 .
$$

It follows from (15) that

$$
j_{\nu}=\nu+O\left(\nu^{1 / 2}\right) \quad \text { for large } \nu .
$$

As an example, the bound for $\nu=0$ is given by $\sqrt{2}+1=2.4142$ in comparison with the true value 2.4048 . 
Acknowledgement. I am indebted to a referee for some improvements in the presentation of this paper.

School of Mathematics and Computer Science

University College of North Wales

Bangor, Gwynedd LL57 2UW, Wales

1. J. Irving \& N. Mullineux, Mathematics in Physics and Engineering, Academic Press, New York, 1959, p. 388.

2. J. IRVING \& N. Mullineux, Loc. cit., p. 39.

3. G. N. Watson, A Treatise on the Theory of Bessel Functions, The University Press, Cambridge, 1944, p. 98.

4. G. N. Watson, Loc. cit., p. 486. 